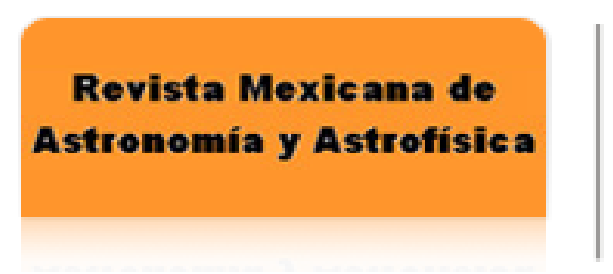

Revista Mexicana de Astronomía y Astrofísica ISSN: 0185-1101

rmaa@astroscu.unam.mx

Instituto de Astronomía

México

Raga, A. C.; Cantó, J.; De Colle, F.; Esquivel, A.; Kajdic, P.; Rodríguez-González, A.; Velázquez, P. F.

RADIATIVE JETS FROM VARIABLE SOURCES

Revista Mexicana de Astronomía y Astrofísica, vol. 36, 2009, pp. 186-192

Instituto de Astronomía

Distrito Federal, México

Available in: http://www.redalyc.org/articulo.oa?id=57115743027

How to cite

Complete issue

- More information about this article

Journal's homepage in redalyc.org

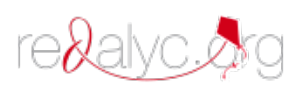

Scientific Information System Network of Scientific Journals from Latin America, the Caribbean, Spain and Portugal Non-profit academic project, developed under the open access initiative 


\title{
RADIATIVE JETS FROM VARIABLE SOURCES
}

\author{
A. C. Raga,${ }^{1}$ J. Cantó, ${ }^{2}$ F. De Colle, ${ }^{3}$ A. Esquivel,${ }^{1}$ P. Kajdic, ${ }^{4}$ A. Rodríguez-González, ${ }^{1}$ P. F. Velázquez ${ }^{1}$
}

\section{RESUMEN}

Presentamos una serie de modelos que exploran distintos aspectos de la formación de superficies de trabajo en jets HH. Estas superficies de trabajo pueden estar en la cabeza del jet (como resultado del "encendido" del jet) o dentro del cuerpo del jet (como resultado de una variabilidad de la eyección). Exploramos el efecto de tener un flujo cónico de distintos ángulos de apertura, y el efecto de tener una velocidad de eyección que varía a través de la sección del jet. También ilustramos las diferencias que se obtienen al variar la resolución de las simulaciones, y al cambiar de descripciones 2D (axisimétricas) a 3D del flujo. Finalmente, describimos el efecto de un campo magnético toroidal sobre las superficies de trabajo de un jet.

\section{ABSTRACT}

We present a series of numerical simulations which explore different aspects of the formation of working surfaces in HH jets. These working surfaces can be at the head of the jet (resulting from a "turning on" of the ejection) or within the body of the jet (resulting from a time-variability of the ejection). We explore the effect of having a conical outflow of different opening angles and the effect of having a non-top hat ejection velocity cross section. We also illustrate the differences that are obtained by varying the resolution of the simulations, and by changing from 2D (axisymmetric) to 3D descriptions of the flow. Finally, we describe the effect of a toroidal magnetic field on the working surfaces of the jet.

Key Words: ISM: Herbig-Haro objects — ISM: jets and outflows — ISM: kinematics and dynamics — stars: pre-main sequence - stars: winds and outflows

\section{INTRODUCTION}

Dopita, Schwartz, \& Evans (1982) interpreted the HH46/47 system as a "starting jet" flow, in which a collimated outflow is more or less suddenly "turned on", and then pushes a "working surface" into the surrounding environment. Over the years, this idea has become quite widely accepted as an explanation for the bow shock-like features seen in some Herbig-Haro (HH) flows.

Raga et al. (1990) suggested that the structures along $\mathrm{HH}$ jets might be the result of a timevariability in the ejection. This is an extension to a multiple-starting jet scenario, in which a series of outflow episodes drive "working surfaces" into the body of the previously ejected material, or into the surrounding environment.

In the last two decades, there have been a number of papers describing analytic models (e.g., Raga \&

\footnotetext{
${ }^{1}$ Instituto de Ciencias Nucleares, Universidad Nacional Autónoma de México, Apdo. Postal 70-543, 04510 D.F., México (raga, esquivel, ary, pablo@nucleares.unam.mx).

${ }^{2}$ Instituto de Astronomía, Universidad Nacional Autónoma de México, Apdo. Postal 70-264, 04510 D.F., México.

${ }^{3}$ Dublin Institute for Advanced Studies, 31 Fitzwilliam Place, Dubln 4, Ireland (fdc@cp.dias.ie).

${ }^{4}$ Instituto de Geofísica, Universidad Nacional Autónoma de México, 04510 D. F., México (primoz@astroscu.unam.mx).
}

Kofman 1992; Cantó, Raga, \& D'Alessio 2000) and numerical simulations (for example, 1D: Hartigan \& Raymond 1993; Massaglia, Mignone, \& Bodo 2005; 2D: Biro \& Raga 1994; Suttner et al. 1997; 3D: de Gouveia Dal Pino \& Benz 1994; Suttner et al. 1997; Rosen \& Smith 1993; Raga et al. 2004) of jets with variable ejection. The problem of magnetized jets from variable sources has also been studied (see, e.g., Cerqueira \& de Gouveia Dal Pino 2001; Stone \& Hardee 2000; Gardiner et al. 2000).

We do not present a review of the existing literature on the subject, which not only covers the effect of variabilities in the (modulus of the) ejection velocity, but also covers the effect of variabilities in the ejection direction (as a result of a precession or of an orbital velocity of the source, e.g., Masciadri \& Raga 2002). Instead, in our paper we describe a set of new models of variable jets.

This set of models (described in $\S 2$ ) is homogeneous in the sense that all models (except one) have been run with the same code, and that they all share the same treatment of the cooling function and the atomic/ionic physics. With these models we first explore the dynamics of the leading working surface caused by a sudden "turning on" of the jet flow (see $\S 3)$. With these models we illustrate the effect of 
VARIABLE JETS

TABLE 1

PARAMETERS OF THE JET MODELS

\begin{tabular}{lcccccccccc}
\hline Model & $v_{0}$ & $\begin{array}{c}r_{j} \\
{\left[\mathrm{~km} \mathrm{~s}^{-1}\right]}\end{array}$ & $\begin{array}{c}n_{j} \\
{\left[10^{15} \mathrm{~cm}\right]}\end{array}$ & $\begin{array}{c}n_{a} \\
{\left[\mathrm{~cm}^{-3}\right]}\end{array}$ & $\begin{array}{c}v_{c} / v_{e} \\
{\left[{ }^{\circ}\right]}\end{array}$ & $\begin{array}{c}\tau \\
{[\mathrm{yr}]}\end{array}$ & $\begin{array}{c}\Delta v \\
{\left[\mathrm{~km} \mathrm{~s}^{-1}\right]}\end{array}$ & $r_{j} / \Delta x$ & $2 / 3 \mathrm{D}^{\mathrm{a}}$ \\
\hline M1 & 200 & 7.0 & 1000 & 50 & 1.0 & 0 & $\ldots$ & $\ldots$ & 71 & $2 \mathrm{D}$ \\
M2 & 200 & 7.0 & 1000 & 50 & 1.0 & 0 & $\ldots$ & $\ldots$ & 71 & $3 \mathrm{D}$ \\
M3 & 200 & 7.0 & 1000 & 50 & 2.0 & 0 & $\ldots$ & $\ldots$ & 71 & $2 \mathrm{D}$ \\
M4 & 200 & 7.0 & 1000 & 50 & 2.0 & 0 & $\ldots$ & $\ldots$ & 71 & $3 \mathrm{D}$ \\
M5 & 200 & 7.0 & 1000 & 200 & 1.0 & 0 & $\ldots$ & $\ldots$ & 18 & $2 \mathrm{D}$ \\
M6 & 200 & 7.0 & 1000 & 200 & 1.0 & 5 & $\ldots$ & $\ldots$ & 9 & $3 \mathrm{D}$ \\
M7 & 200 & 7.0 & 1000 & 200 & 1.0 & 5 & $\ldots$ & $\ldots$ & 9 & $2 \mathrm{D}$ \\
M8 & 200 & 7.0 & 1000 & 200 & 1.0 & 5 & $\ldots$ & $\ldots$ & 18 & $2 \mathrm{D}$ \\
M9 & 200 & 7.0 & 1000 & 200 & 1.0 & 15 & $\ldots$ & $\ldots$ & 9 & $3 \mathrm{D}$ \\
M10 & 200 & 7.0 & 1000 & 200 & 1.0 & 15 & $\ldots$ & $\ldots$ & 9 & $2 \mathrm{D}$ \\
M11 & 200 & 7.0 & 1000 & 200 & 1.0 & 15 & $\ldots$ & $\ldots$ & 18 & $2 \mathrm{D}$ \\
M12 & 200 & 7.0 & 1000 & 100 & 1.0 & 0 & 30 & 30 & 18 & $2 \mathrm{D}$ \\
M13 & 200 & 7.0 & 1000 & 100 & 1.0 & 5 & 30 & 30 & 18 & $2 \mathrm{D}$ \\
M14 & 200 & 7.0 & 1000 & 100 & 2.0 & 0 & 30 & 30 & 18 & $2 \mathrm{D}$ \\
M15 & 300 & 2.0 & 100 & 10 & 1.0 & 0 & 20 & 150 & 80 & $2 \mathrm{D}$ \\
\hline
\end{tabular}

${ }^{\mathrm{a}}$ All of the $3 \mathrm{D}$ simulations have a sinusoidal density perturbation of $5 \%$ half-amplitude and $r_{j} / 2$ wavelength in the injection density cross-section.

${ }^{\mathrm{b}}$ Model M15 has been computed with and without a toroidal magnetic field (equation 5. with $B_{0}=5 \mu \mathrm{G}$ and with $\left.B_{0}=0\right)$.

having an inflow with different initial opening angles, and with a "centre-to-edge" ejection velocity dependence in the ejection cross section. We also illustrate the differences which are obtained with $2 \mathrm{D}$ (axisymmetric) and with 3D simulations. We then explore models with internal working surfaces resulting from an ejection velocity time-variability $(\S 4)$, and illustrate the effects of a non-zero ejection opening angle and a centre-to-edge ejection velocity profile. Finally $(\S 4.2)$, we illustrate the effect of a toroidal magnetic field on the structure of internal working surfaces.

\section{THE NUMERICAL SIMULATIONS}

We present a series of 2D (axisymmetric) and 3D simulations of radiative jets. The jets move into a homogeneous environment, and have initially tophat and time-independent density and temperature cross sections.

The jet velocity, however, is allowed to have a sinusoidal time variability (with an on-axis mean velocity $v_{0}$, half-amplitude $\Delta v$ and period $\tau$ ), and a quadratic, centre-to-edge dependence with a $\left(v_{c} / v_{e}\right)$ velocity contrast. The velocity with which the jet is ejected is therefore given by:

$$
v_{j}(r, t)=\left[v_{0}+\Delta v \sin \left(\frac{2 \pi t}{\tau}\right)\right] \times
$$

$$
\left[1-\left(1-\frac{v_{e}}{v_{c}}\right)\left(\frac{r}{r_{j}}\right)^{2}\right] .
$$

The jet is ejected as a conical outflow of half-opening angle $\alpha$. Therefore, the axial ejection velocity is

$$
v_{x}(r, t)=\sqrt{1-\left(\frac{r \sin \alpha}{r_{j}}\right)^{2}} v_{j}(r, t),
$$

and the radial component (i. e., the component perpendicular to the jet axis) of the ejection velocity is

$$
v_{r}(r, t)=\left(\frac{r \sin \alpha}{r_{j}}\right) v_{j}(r, t) .
$$

A series of 15 models were run, all of them sharing an injection temperature $T_{j}=1000 \mathrm{~K}$ and an environmental temperature $T_{e}=100 \mathrm{~K}$. The other parameters for the jet inflow and for the (initially homogeneous) environment are listed in Table 1. Models M1-M14 were computed with 2D (axisymmetric) and 3D versions of the yguazú-a code (Raga, Navarro-González, \& Villagrán-Muniz 2000). The version of the code which was used integrates the gasdynamic equations together with a single rate equation for neutral $\mathrm{H}$, and uses the temperature, density and $\mathrm{H}$ ionisation fraction to compute a parametrised 


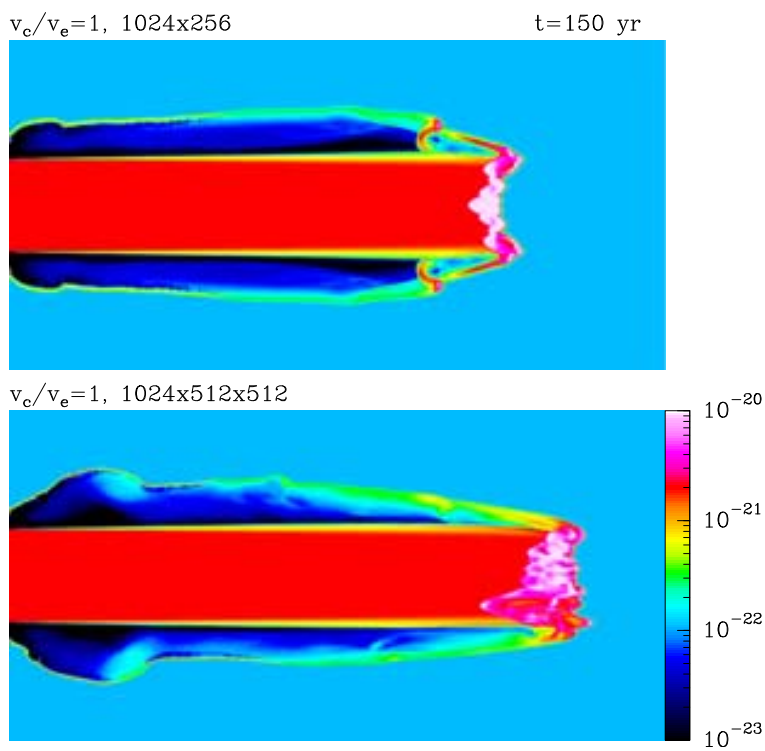

Fig. 1. Axisymmetric (top, model M1) and 3D (bottom, model M2) simulations of a "top hat" starting jet. The graphs show the density stratification (given in $\mathrm{g} \mathrm{cm}^{-3}$ by the bar on the bottom right) obtained after a $t=150 \mathrm{yr}$ time-integration. The labels on the top left of each panel give the equivalent resolution in pixels of a uniform grid. The displayed domain has an axial extent of $10^{17} \mathrm{~cm}$. The results from the axisymmetric simulation (M1, top) have been reflected on the symmetry axis.

cooling function (as described by Raga \& Reipurth 2004). A seed electron density (assumed to arise from singly ionised C) is assumed, and the initial configuration of the flow (both for the jet and the environment) is otherwise neutral. In all cases, a 6-level, binary adaptive grid was used.

Model M15 (see Table 1) was computed with the uniform grid code described by De Colle \& Raga (2006), and the same setup (described above) was used for computing the radiative cooling. For all models, outflow conditions were applied on all boundaries except on the $x=0$ boundary (where the jet is injected at cylindrical radii $r \leq r_{j}$ and a reflection condition is imposed for $r>r_{j}$ ) and on the symmetry axis (in the case of the $2 \mathrm{D}$, axisymmetric simulations).

\section{THE LEADING WORKING SURFACE}

\subsection{Top hat jets}

The most simple possible ejection timevariability in a jet flow is the so-called "starting jet" problem, in which a jet is suddenly "turned on" within a stationary environment.
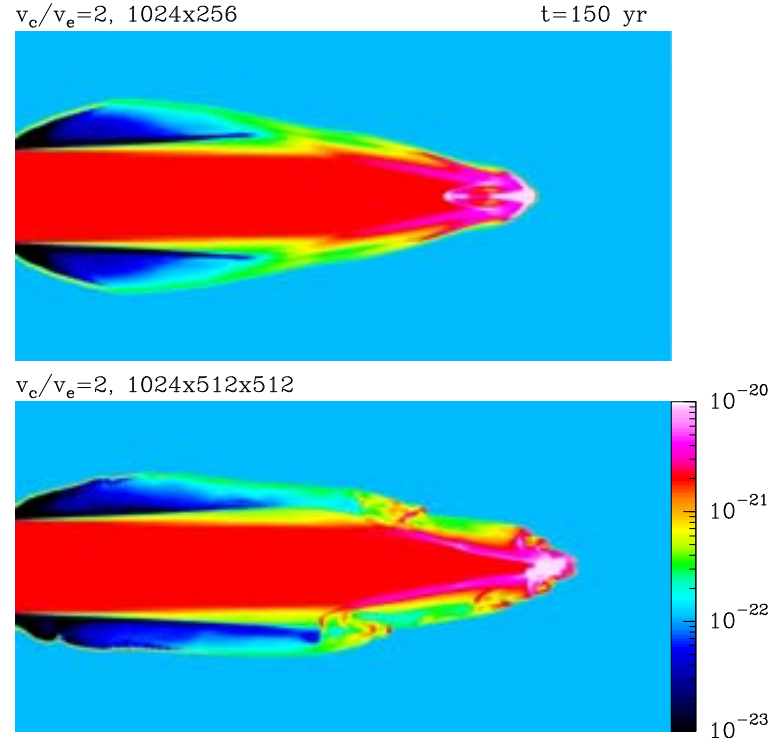

Fig. 2. Axisymmetric (top, model M3) and 3D (bottom, model M4) simulations of a starting jet with a quadratic initial velocity cross section, with a centre-to-edge contrast of $v_{c} / v_{e}=2$. The displayed domain has an axial extent of $10^{17} \mathrm{~cm}$.

Figure 1 shows numerical experiments of a jet (suddenly "turned on" at $t=0$ ) with a "top hat" initial cross section, computed in 2 and 3D (models M1 and M2 of Table 1). The 2D and 3D models produce qualitatively similar results, but the $3 \mathrm{D}$ model has a much thicker region in between the jet and bow shocks. This region is less dense and axially more extended in the the 3D simulation (model M2).

\subsection{Non-top hat jets}

Figure 2 shows the results obtained in 2D (model M3) and 3D (model M4) for a non-top hat injection velocity cross section with $v_{c} / v_{e}=2$ (see $\S 2$ and Table 1). In model M3 (2D), we see the production of a dense "nose cone", into which converges the material passing through a oblique jet shock (such structures have been studied, e.g., by Raga, Cantó, \& Cabrit (1998).

The production of a "nose cone" to some extent survives in the 3D simulation (model M4, see Figure 2 and Table 1). However, the nose cone is somewhat wider and less axially extended than in the $2 \mathrm{D}$ (axisymmetric) simulation.

\subsection{Conical jets}

Another possibility is to consider jets with initially conical cross sections (but with otherwise "top 

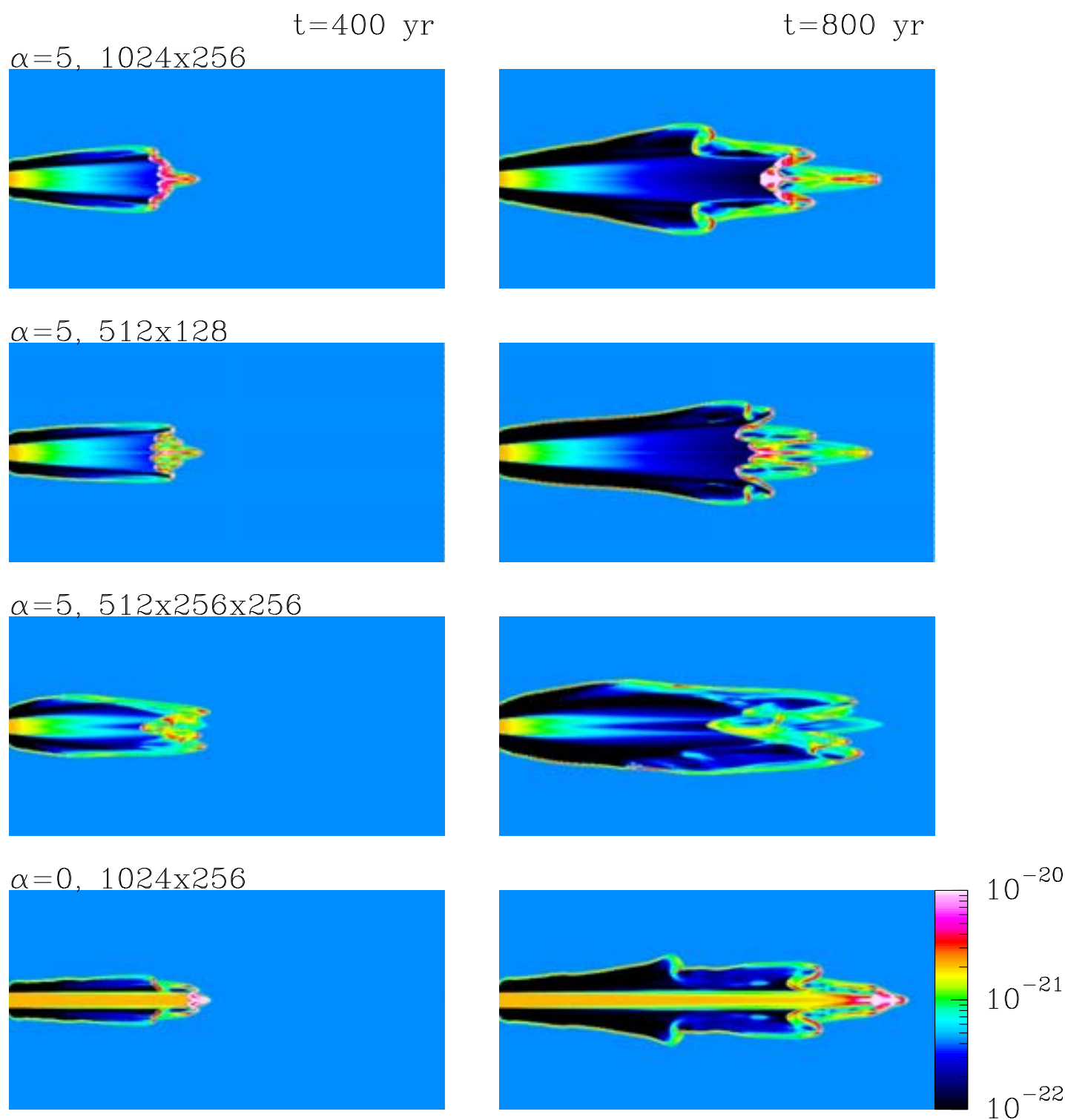

Fig. 3. Mid-plane density stratifications obtained for time integrations $t=400$ (left) and 800 yr (right) for models M5 (bottom, with zero initial opening angle) through M8 (top). Models M6-M8 have an $\alpha=5^{\circ}$ initial half-opening angle. All of the models are axisymmetric except M7 (second from bottom, also see Table 1). The displayed domain has an axial extent of $4 \times 10^{17} \mathrm{~cm}$.

hat" characteristics). Figure 3 shows a comparison between a $2 \mathrm{D}$, top hat jet (model M5, bottom frames) and 3 models with the same parameters but an $\alpha=5^{\circ}$ initial half-opening angle (models M6-M8, see Table 1).

The 3D model M6 shows a broad leading working surface, which develops an axially extended, complex shock structure. The axisymmetric, $\alpha=5^{\circ}$ simulations (models M7 and M8, see Figure 3 and Table 1) develop an axial protrusion, which is ab- sent in the $3 \mathrm{D}$ simulations, and should in principle be regarded as a feature that would never appear in astrophysical jets (in which substantial perturbations from axisymmetry are many times observed). This axial feature appears in the two spatial resolutions explored by the 2D simulations (models M7 and M8, see Table 1 and Figure 3).

Figure 4 again shows the top hat jet M5 (see Table 1) and a group of 3 models (M9-M11) with an $\alpha=15^{\circ}$ initial half-opening angle (see Table 1 ). The 

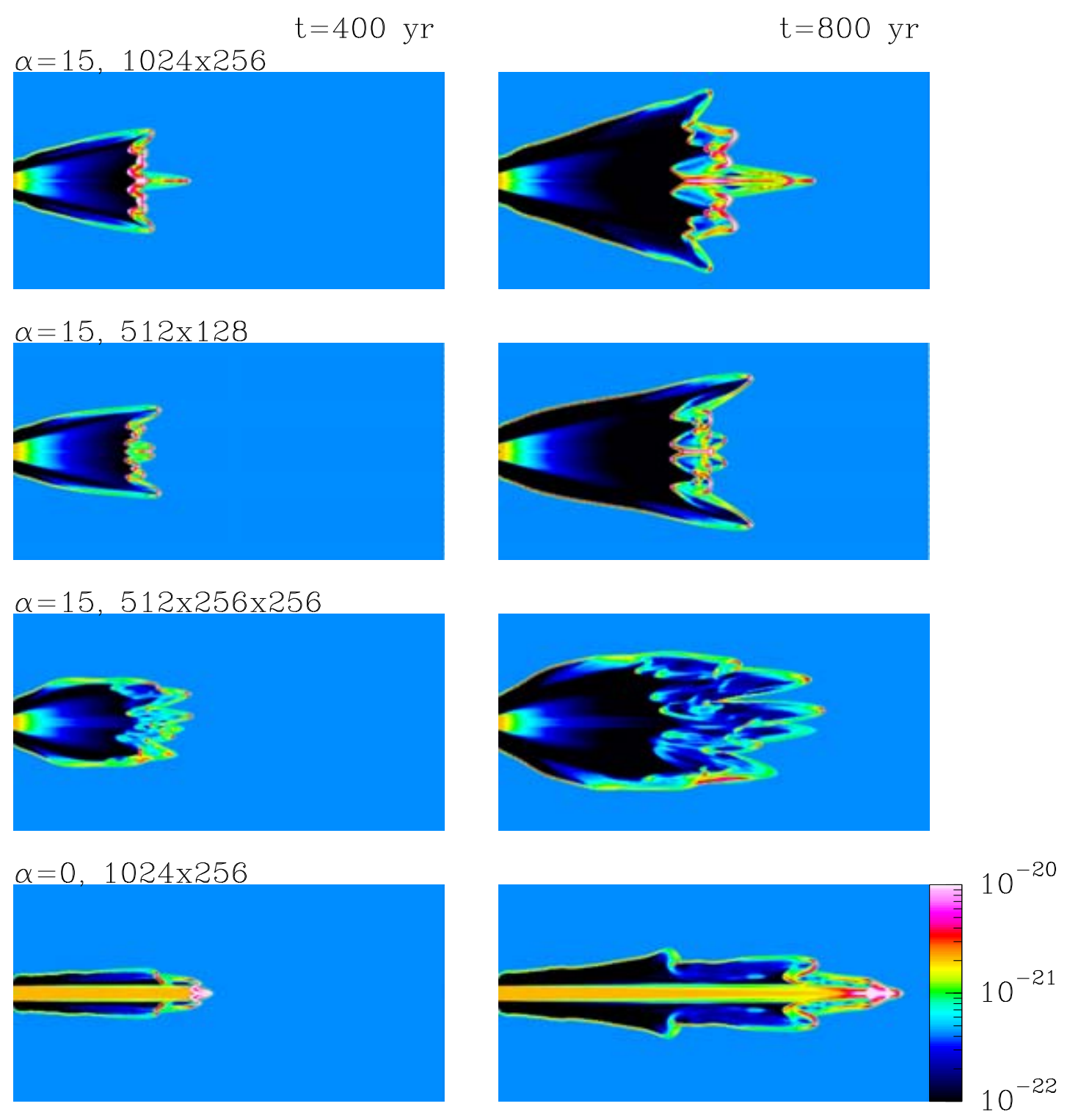

Fig. 4. Mid-plane density stratifications obtained for time integrations $t=400$ (left) and 800 yr (right) for models M5 (bottom, with zero initial opening angle), M9, M10 and M11 (top). Models M8-M10 have an $\alpha=15^{\circ}$ initial half-opening angle. All of the models are axisymmetric except M9 (second from bottom, also see Table 1). The displayed domain has an axial extent of $4 \times 10^{17} \mathrm{~cm}$.

3D model M9 shows a leading working surface which fragments into a number of "fingers" which propagate into the surrounding environment. The axisymmetric models (M10 and M11) develop an outer ring structure, which propagates into the environment approximately in the direction defined by the outer walls of the initial cone. Quite notably, the higher resolution 2D model (M11) develops an axial, collimated feature which does not appear in the lower resolution 2D model (M10). Actually for longer in- tegration times (than the ones shown in Figure 4), model M11 also develops an axial protrusion.

\section{INTERNAL WORKING SURFACES \\ 4.1. Non-magnetized jets}

Figure 5 shows the results obtained for jets with a sinusoidal ejection velocity variability (see equation 1). Model M12 has a top-hat injection cross section. Model M13 has a conical injection (of half-opening angle $\alpha=5^{\circ}$ ), and model M14 has a 


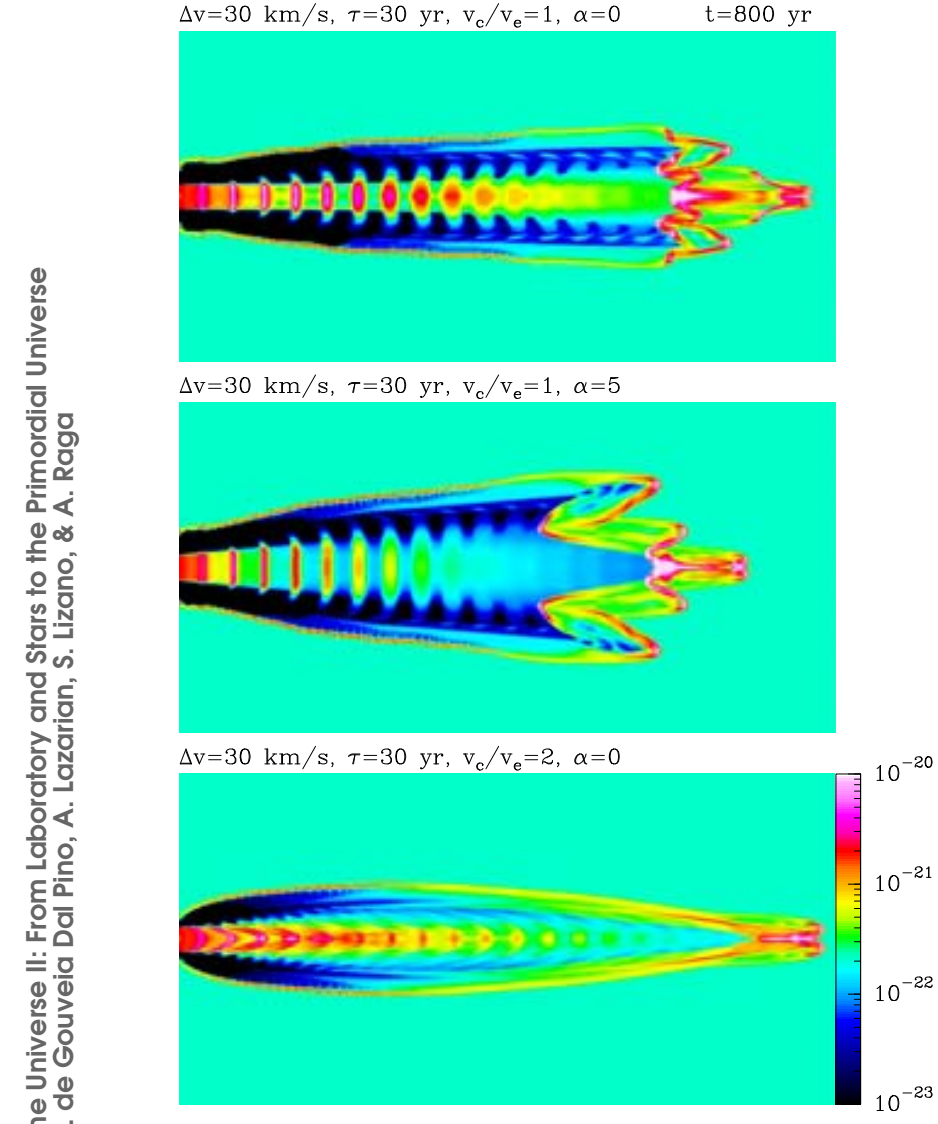

Fig. 5. Density stratifications obtained after a $t=800 \mathrm{yr}$ time-integration for models M12 (top) through M14 (bottom, see Table 1). These models have a sinusoidal ejection velocity variability, and explore the effects of having a top-hat, zero initial opening angle (M12, top), an $\alpha=5^{\circ}$ half-opening angle (M13, centre) and a quadratic centre-to-edge initial velocity profile (with a $v_{c} / v_{e}=2$ contrast, M14, bottom). The displayed domain has an axial extent of $4 \times 10^{17} \mathrm{~cm}$.

$v_{c} / v_{e}=2$ centre-to-edge velocity ratio. This figure illustrates the strong effect on the morphology of the internal working srufaces produced by the characteristics of the injection cross section.

\subsection{Magnetized jets}

If the magnetic field within the jet beam is strong enough, the magnetic pressure (or pinch force) $f_{m}$ in the radiatively cooled internal working surface will dominate over the gas pressure $f_{p}$. For a working surface shock with an Alfvénic Mach number $M_{A} \ll M_{w}^{2}$ (where $M_{w}=v / c_{w}$ is the Mach number calculated with the shock velocity $v$ and the sound speed $c_{w}$ of the cooled internal working surface), De Colle, Raga, \& Esquivel (2008) derived the scaling
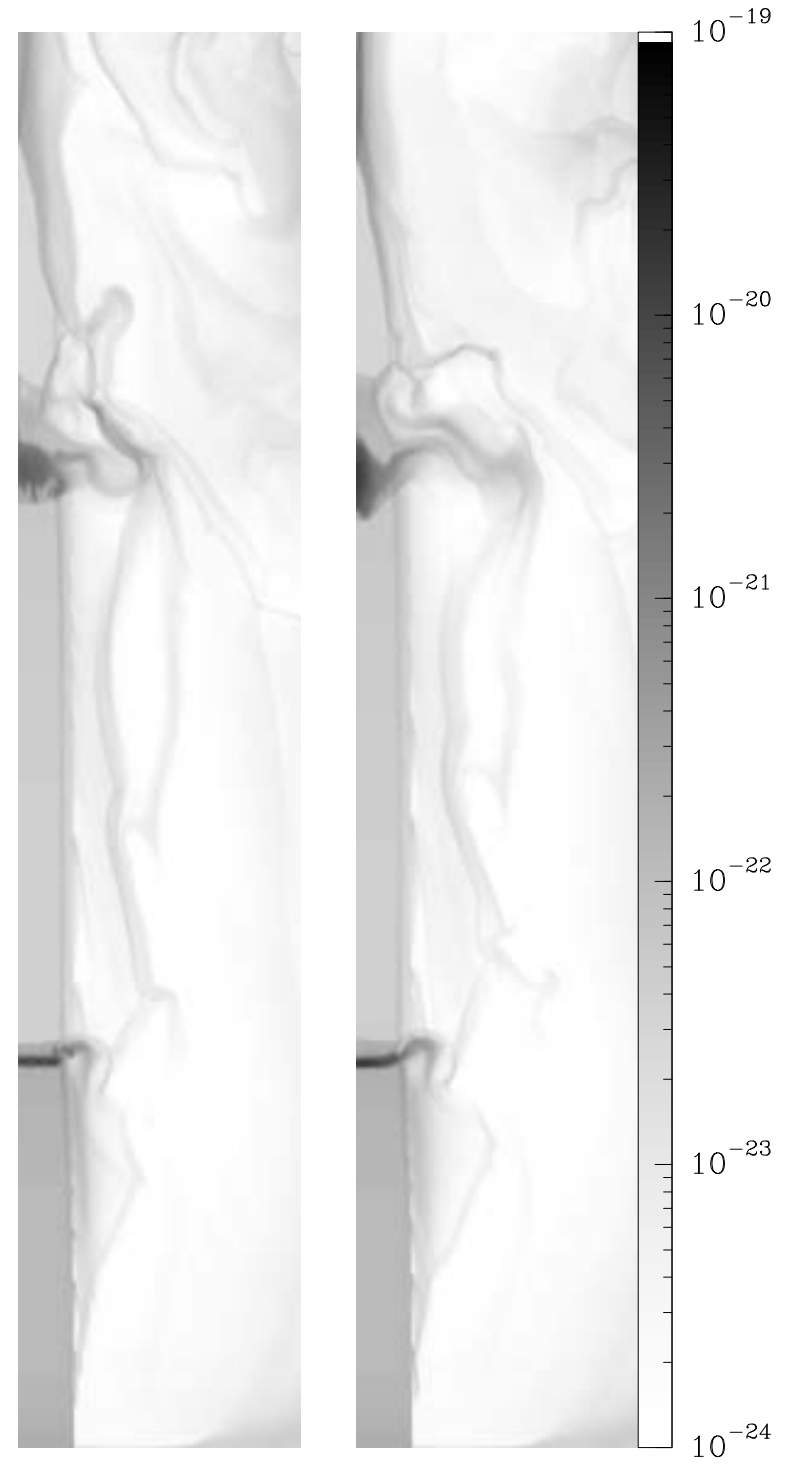

Fig. 6. Density stratifications (given in $\mathrm{g} \mathrm{cm}^{-3}$ by the bar on the right) obtained from model M15, for a $t=$ 90 yr time integration. The results obtained with $B_{0}=0$ (left) and $B_{0}=5 \mu \mathrm{G}$ (right, see equation 5) are shown. The axial (vertical) extent of the displayed domain is of $2 \times 10^{16} \mathrm{~cm}$.

law

$$
\frac{f_{m}}{f_{p}} \sim \frac{M_{w}^{2}}{M_{A}} .
$$

In other words, for a jet beam with equipartition (for which we would have $M_{A} \sim M_{w}$ ), and an internal working surface with $M_{w} \sim 10$, we would have $f_{m} / f_{p} \sim 10$. Therefore, the magnetic pinch force would dominate over the gas pressure (by an order 
of magnitude) in the cooled material within the internal working surface.

In Figure 6, we show the results from a nonmagnetized and a magnetized, variable jet simulation. This figure shows model M16 (see Table 1), with an injection toroidal magnetic field configuration

$$
B(r)=B_{0} \frac{r}{r_{j}},
$$

with $B_{0}=0$ (left frame of Figure 6 ), and $B_{0}=5 \mu \mathrm{G}$ (right frame). It is clear that the internal working surfaces of the magnetized jet model develop a strong, axial focussing which is absent in the nonmagnetized jet model.

\section{CONCLUSIONS}

We have presented a set of 15 radiative jet simulations, illustrating a series of points:

- pairs of models with the same parameters and resolutions (M1 and M2, M3 and M4, M6 and M7, M9 and M10) show the differences that are obtained if one goes from 2D (axisymmetric) to 3D simulations (see Figures 1-4),

- pairs of axisymmetric models with the same parameters but different resolutions (M7 and M8, M10 and M11) show the effect of changing the numerical resolution of the simulations (see Figures 34),

- the effect on the leading working surface of having a non-top hat ejection velocity cross section has been explored (Figure 2),

- the effect on the leading working surface of having an initially conical ejection with different values of the half-opening angle $\alpha$ has been explored (see Figures 3-4),

- the effect on internal working surfaces (resulting from an ejection velocity time-variability) of a non-zero initial opening angle and a non-top hat initial velocity cross section has been explored (Figure 5),

- the effect on internal working surfaces of having a magnetized jet beam with a toroidal magnetic field configuration has been explored (Figure 6).

These simulations are similar to many calculations in the literature, but they might be useful as a simple guide on what effects are to be expected for different possible configurations of "starting jet" or "variable jet" models.

Clearly, the exercise of trying out different possible injection variabilities is infinite, and one can question to what extent is it worth to keep on computing such models. One possible way to make it worthwhile is to attempt to model particular $\mathrm{HH}$ outflow systems. The models in this paper may provide a guideline of what parameters to explore in order to reproduce a chosen $\mathrm{HH}$ object.

Modelling specific systems provides a way to determine which are the possible characteristics of the ejection variability implied by the observed structures along an $\mathrm{HH}$ flow. This kind of exercise is useful for providing constraints on future models of the production of jets from young star/circumstellar disk models.

\section{REFERENCES}

Biro, S., \& Raga, A. C. 1994, ApJ, 434, 221

Cantó, J., Raga, A. C., \& D'Alessio, P. 2000, MNRAS, 313,656

Cerqueira, A. H., \& de Gouveia Dal Pino, E. M. 2001, ApJ, 560, 779

Dopita, M. A., Schwartz, R. D., \& Evans, I. 1982, ApJ, 236, L73

De Colle, F., \& Raga, A. C. 2006, A\&A, 449, 1061

De Colle, F., Raga, A. C., \& Esquivel, A. 2008, ApJ, 689, 302

de Gouveia Dal Pino, E. M., \& Benz, W. 1994 ApJ, 435, 261

Gardiner, T. A., Frank, A., Jones, T. W., \& Ryu, D. 2000, ApJ, 530, 834

Hartigan, P., \& Raymond, J. C. 1993, ApJ, 409705

Masciadri, E., \& Raga, A. C. 2002, 568, 733

Massaglia, S., Mignone, A., \& Bodo, G. 2005, A\&A, 442, 549

Raga, A. C. Cantó, J., Binette, L., \& Calvet, N. 1990, ApJ, 364, 601

Raga, A. C., Cantó, J., \& Cabrit, S. 1998, A\&A, 332, 714

Raga, A. C., \& Kofman, L. 1992, ApJ, 386, 222

Raga, A. C., Navarro-González, R., \& Villagrán-Muniz, M. 2000, RevMexAA, 36, 67

Raga, A. C., \& Reipurth, B. 2004, RevMexAA, 40, 15

Raga, A. C., Riera, A., Masciadri, E., Beck, T., Aspin, C., Böhm, K. H., \& Binette, L. 2004, AJ, 127, 1081

Rosen, A., \& Smith, M. D. 2003, MNRAS, 343, 81

Stone, J. M., \& Hardee, P. E. 2000, ApJ, 540, 192

Suttner, G., Smith, M. D., Yorke, H. W., \& Zinnecker, H. 1997, A\&A, 318, 595 